\title{
Optimum time interval for intertwin delivery for extreme prematurity in DCDA twin pregnancy. A case report and a literature review.
}

\author{
Prabha Sinha', Olakanmi Joseph ${ }^{1}$, Aoun Hakmi \\ ${ }^{1}$ Sidra Medicine and Research Centre, Doha, Qatar \\ ${ }^{2}$ Tawam Hospital, Al Ain, Abu Dhabi, UAE
}

\section{Correspondence}

Prabha Sinha MBBS, DRCOG, MRCPI, FRCOG, Senior Attending Physician, Sidra Medicine, t. +974 40036860 ,

m. +974 55907685, PO BOX 26999 Doha, Qatar, E mail: psinha@sidra.org

\section{Abstract}

Due to increased use of ART delayed twin interval delivery has become a common occurrence. Multiple pregnancy is associated with high incidence of premature labour. Prematurity is the leading cause of neonatal morbidity and mortality all over the world as premature babies are at a greater risk of dying and has other health issues. Delivery of the presenting fetus usually followed by delivery of the second fetus shortly thereafter. There is no study available for the management of multifetal pregnancy, where one twin had delivered very prematurely and the time interval of the delivery for subsequent fetus. Conservation of pregnancy has good outcome when properly managed with careful surveillance. Survival rate increases by approximately $10 \%$ every week conserved in utero. Reservation persist in anticipation of increased maternal morbidity and mortality due to infection after conservative management.Time interval remains a dilemma in extreme prematurity.

Key words: multiple pregnancy, prematurity, intertwin delivery, PPROM, Chorioamnionitis

\section{Introduction}

In the last two decades, the number of multiple pregnancies has increased because of Artificial Reproductive Technology (ART) ${ }^{1}$.As a result, preterm labour in the second trimester and the PPROM of the presenting fetus have now been commonly seen.

Prematurity is the leading cause of neonatal morbidity and mortality all over the world ${ }^{2,3}$.Multiple pregnancy is associated with high incidence of pre- mature labour ( $4 \%$ at 30 and up to $16 \%$ before 34 weeks of gestation).

The babies born at 22 weeks has survival rate of almost zero which increases to $19 \%$ at 23 weeks, $40 \%$ at $24,66 \%$ at 25 and almost $77 \%$ at 26 weeks. Survival rate increases by approximately $10 \%$ every week conserved in utero if born around 23 weeks and $16 \%$ per week if born around 25 weeks. Children born before 27 weeks has high degree of disability(at 
26 weeks 45\%) and serious cognitive impairment ${ }^{4.5}$. Preterm babies are also at a greater risk of dying, and has other health issues such as respiratory, GI, CNS, hearing, vision, immunological, as well as other late neurodevelopmental complications.

In multiple pregnancy delivery of the presenting fetus usually followed by delivery of the second fetus or fetuses shortly thereafter. Traditionally 20-30 minutes is used as the upper limit for the delivery of second twins. Some study suggests longer intertwin birth time interval is associated with a continuously slow decline in umbilical cord PH.Risks of suspicious CTG/ fetal compromise and acidosis in the second twin are high when the twin-to-twin delivery interval is beyond 30 minutes. These effects are anticipated as uterine volume decreases resulting in placental separation or cord compression impairing the blood flow to the placenta and the remaining fetus. However, other study has highlighted the small differences in $\mathrm{PH}$ is not significant enough to impact on clinical management ${ }^{6.7 .8}$. Recently published retrospective study of 713 twins, 24 weeks and above over a 10 years period on timing for intertwin delivery has not shown any difference in the Cord $\mathrm{PH}$ and Apgar score after 30 mintues $^{9}$.As there are two placental masses in DCDA twins effect in the remaining fetus in the presence of intact membrane logically is minimal thereby effect on the fetus is minimal. Perhaps that justify conservation of remaining twins in very premature fetuses without any problems. Available data does not suggest increased morbidity by conserving the pregnancy apart from infective morbidities for mother and the baby.

There is no study or guidelines are available for the management of multifetal pregnancy, where one twin had delivered very prematurely and the time interval for the delivery of subsequent fetus in DCDA twin gestation. There are several cases have been reported with good outcomes, but reservation persist in anticipation of increased maternal morbidity and even maternal mortality due to infection after conservative management for the remaining fetus.

In cases where one of the fetuses can be lost and the remaining one/s retained can be continued with good outcome when properly managed with careful surveillance.

We report a case of delayed delivery with an interval of 46 days after the delivery of the first win delivered spontaneously.

\section{Case Report}

A 34 year old, G8P7 had all previous SVD. She had 6 living children out of which 3 of them had history of genetic heart problem. Current pregnancy was conceived with IVF/PGD with DCDA twin. She was booked in the private sector at 10 weeks and was diagnosed to have GDM which was diet controlled. Anatomy USS scan had suggested marginally lowlying placenta for second twins.

She attended delivery room on $24+6 / 52$, with history of a gush of fluid per vagina which was not associated with bleeding or contractions. Her vital signs were normal.

Patient was admitted with confirmed PPROM. Her HB was 9.7gram, WBC 10.5, CRP105, AB+ve, urine dipstick 3+leukocyte, 3+ketons, 2+erythrocyte, urine culture and HVS was negative.

USS suggested 1st twin in breech presentation with fetal part noted to be deeply engaged within the pelvis. Fetal biometry was on 4th centile corresponding to 22 weeks gestation with EFW 490gram.

2nd twin was in transverse lie, fetal biometry on 16th centile corresponding to 23 weeks and with EFW 560gms.

One Placental bulk seen the left anterior, high and another bulk more on posterior and right lateral difficult to rule out low lying placenta because of fetal position.

She was started on erythromycin 500mg QID with betamethasone injection $12 \mathrm{mg}, 2$ doses 
24hours apart and insulin on sliding scale.

Following day,she went into labour and delivered 1st twin vaginally at 03:39 hour, baby boy weight 420 grams with Apgar score of 0 at 1 mint, 5 at 5mint, intubated and admitted to NNU.

The umbilical cord was cut and ligated. Placenta remained retained. After delivery of first twin, membrane of 2nd twin was intact with minimal vaginal bleeding and contractions had subsided. Patient and family was counseled about conservative management due to extreme prematurity. They were told about advantage and disadvantage including risks of maternal and fetal infection, prolonged hospital stays and close surveillance.

They had accepted the plan of conservative management. Cord was hanging outside and was smelly therefore it was cut short, (absorbable suture used) which retrieved up inside the uterus gradually.

Patient was managed with endocrinologist and put on subcutaneous injection insulin to control her

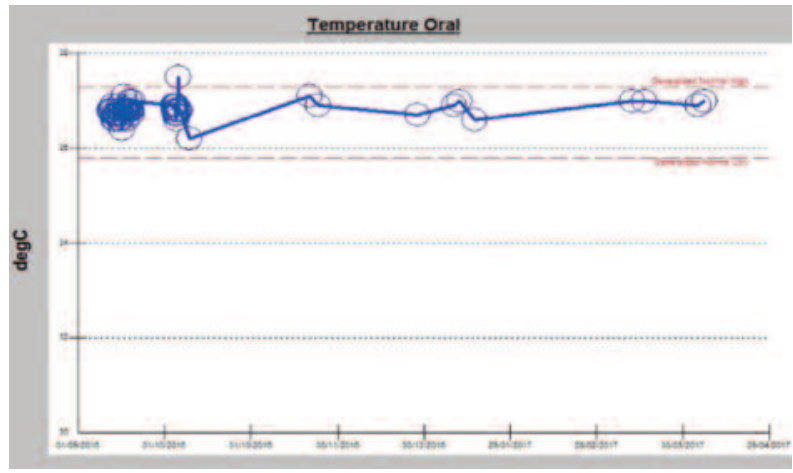

Figure 1. Showing temperature chart

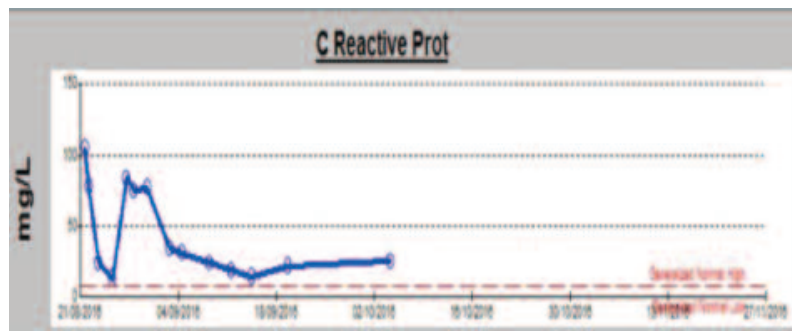

Figure 2. Showing CRP trend blood sugar. Vital signs were checked 6hourly. including temperature (Figure 1), CRP (Figure 2) and WBC (Figure 3) were repeated twice weekly. Fibrinogen (Figure 4) and HVS weekly performed. HVS showed Gardenella vaginalis which was treated by metronidazole IV 500mg TID for 7 days along with Erythromycin.

USS was repeated regularly in FMU which showed progressive fetal growth with normal amniotic fluid, Dopplers. The placenta of 2nd twin was posterior and low lying. In subsequent scans the placenta had moved and was more than $2 \mathrm{~cm}$ away from the internal os. Placenta of first twin was getting thinner (Figure 5).

After nearly 3 weeks, she was discharged home on insulin/metformin and haematinic with instructions to report any fever, uterine contractions or vaginal bleeding/leaking with weekly follow up in the outpatient clinic. After 16 days at 30+ presented to Labour room with uterine contraction. Vaginal assessment confirmed os to be $5 \mathrm{~cm}$ dilated, head at 0 station and well applied. No active bleeding noted. Labour progressed and delivered vaginally 4 hours late, baby boy, weight 1280 gram with Apgar of 8, 9, 10. Both

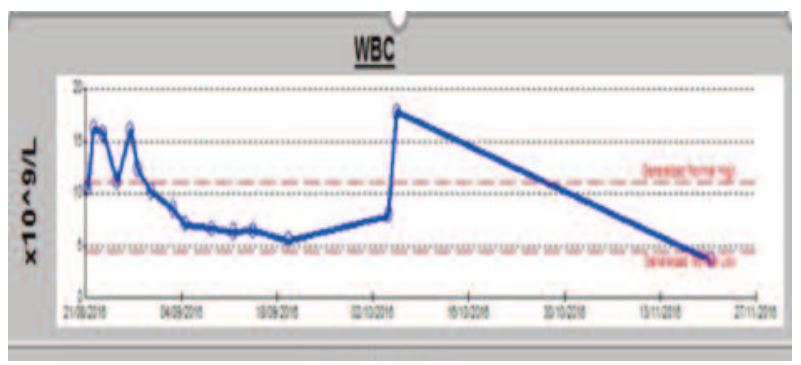

Figure 3. Showing WBC trend.

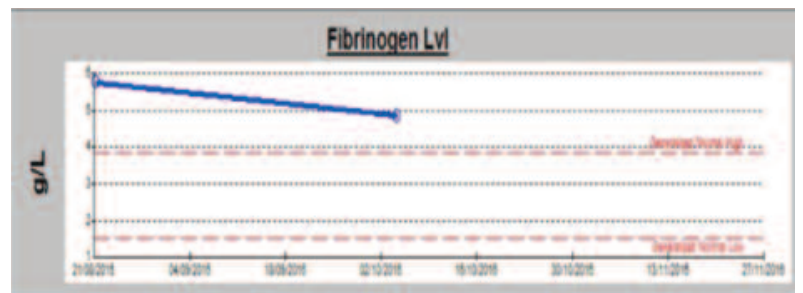

Figure 4. Showing fibrinogen level 
placentas delivered spontaneously but there was missing part of 1st placenta. Exploration of uterus was carried out under GA and placental tissue was removed and sent for histopathology (Figure 6).

Patient had smooth postnatal period and discharged home in good condition.

The management consisted of careful monitoring of both maternal and fetal status. Forty-six days later. Both twins received full resuscitation and immediate life-support intervention. First twin had stormy recovery with IVH type 1 and RDS which was treated successfully. Now twins are 16 months of
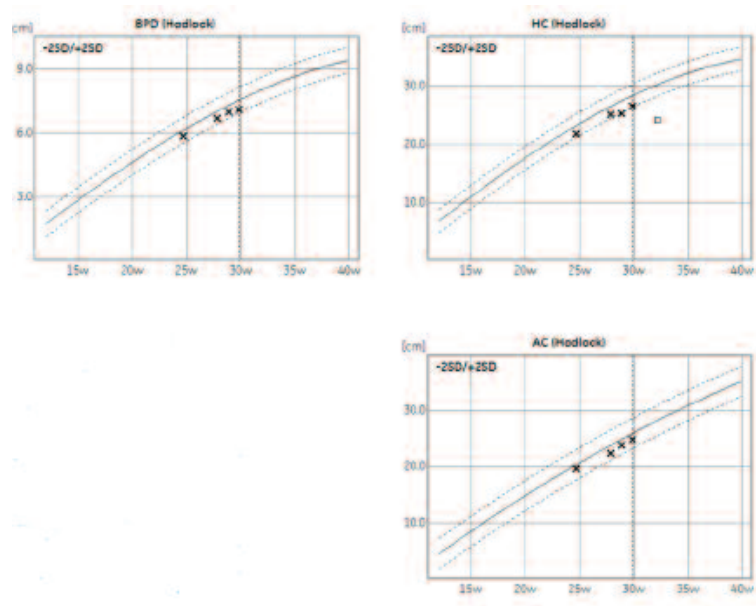

Figure 5. Scan showing growth trend

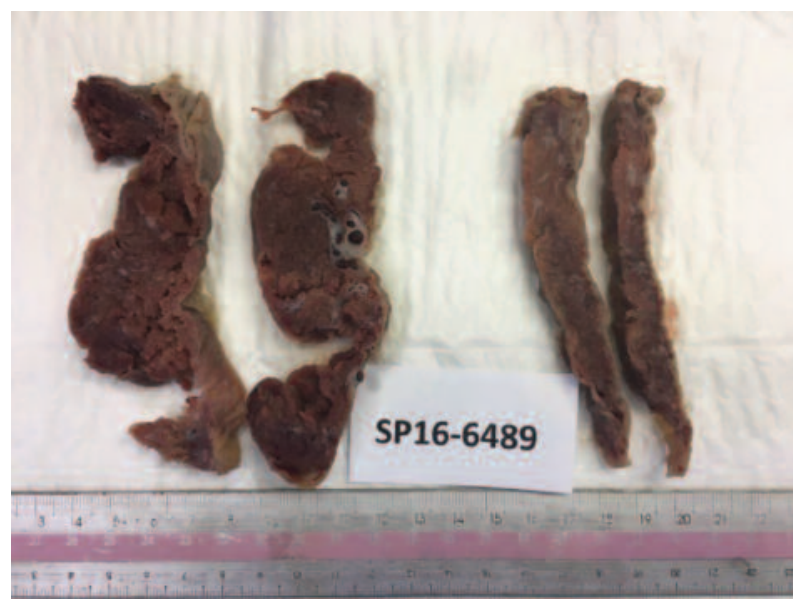

Figure 6. Showing placental histology for twin A and B age and both have normal neuro-development so far.

\section{Discussion}

In dichorionic and dizygotic multiple pregnancy, each fetus has its own placenta, amnion and chorion, therefore conservative management in very premature pregnancy is a viable option. Full counselling including advantage and complications of conservative treatment should be discussed with the patient and her relatives.

Our experience of twin pregnancy with a 46 days' delivery interval illustrates the potential for a satisfactory outcome without cerclage. While the longest known reported intertwin delivery interval with cerclage is 153 days.

Gestational age at delivery is an important factor in neonatal survival as this in most cases is directly related to fetal weight. Prolongation of gestational age and subsequent increase in fetal weight will lead to significant improvement in fetal outcome.10

A delay of two or more days in the delivery of babies born before 30 weeks of gestation is associated with improved infant survival and higher infant birth weight.

Controversies persist on how best to manage delayed interval delivery of remaining fetus/fetuses because such cases are still relatively rare. Efforts should be made to prolong such pregnancy following spontaneous miscarriage or premature birth of one of the fetuses.

Since cases with positive outcomes (including the current report) are more likely to appear in the medical literature than those describing an unsatisfactory result, a publishing bias may favour the former.

This case has raised some key issues in the cause of preterm labour and management of the retained twin. Although the vital signs and the culture results were normal, the significantly raised CRP suggest the possibility of an inflammatory/infective process or caused by tissue damage after delivery of first twins. In the absence of other inflammatory marker and de- 
clining CRPafter therapeutic dose of erythromycin $(500 \mathrm{mg})$ instead of the prophylactic dose $(250 \mathrm{mg})$ may have made a difference in preventing the progress of labour. Furthermore, the subsequent culture of Gardnerella vaginalis and its treatment with IV metronidazole further strengthen the point that actively treating infective process may be the mainstay of treatment required in this type of cases.

Known cause of pre-term labour include idiopathic, infection (40\%), multiple pregnancy, placental abruption, hormonal disruption and others ${ }^{11,12}$

Up to $80 \%$ of women who deliver before 30 weeks gestation have evidence of bacterial infection in the amniotic fluid and/or membrane. ${ }^{13}$

Organisms frequently cultured following preterm delivery include Ureaplasma urealyticum, Mycoplasma hominus, Bacteroides species and Gardnerella vaginalis. When such organisms are found in the amniotic fluid of pregnant women prior to 20 weeks, the pregnancy usually ends 4 to 8 weeks later.

Bacterial vaginosis at 13-16 weeks gestation has a 5-7-fold increased risk of second trimester miscarriage and preterm birth before 34 weeks. ${ }^{14,15}$

In a more focused systematic review and metaanalysis, has suggested clindamycin in women with bacterial vaginosis before 22 weeks gestation which reduces preterm labour by $40 \%$ and late miscarriage by $80 \%{ }^{16}$. Therefore, other than the use of Metronidazole, Clindamycin may be considered as an alternative.

Cerclage has its inherent potential complications and may even be harmful in the presence of undetected /treated infections. In this case Cervix was shutting down itself therefore was not considered.

After reviewing the literature, we suggest conservation of pregnancy for the remaining twins where the following criteria are met.

\section{Inclusion criteria:}

- Twins should be DCDA gestation
- Contraction should be ceased spontaneously

- Cervix should start closing

- Membrane of the remaining twins should be intact

- No obvious signs of infection

- Pregnancy before 28 weeks or before depending on the local Neonatal intensive care unit

- Cevical cerclage may be considered after due consideration

- Parental approval prior to conservation

\section{Exclusion criteria:}

- continuing uterine contraction

- Rupture of the membrane for the second twins

- Any signs of abruptions

- Abnormal fetal heart rate showing signs of compromise

- Fetal anomaly

- Strong evidence of chorioamninitis

Other modalities of management suggested in the literature such as amniocentesis was not done in this case. Although one can understand the rationale for doing amniocentesis, it is important to bear in mind the risk of amniocentesis which may lead to infection and or miscarriage. As such one may suggest that overall it might be better to use other surrogate markers of infection to decide/monitor treatment if the advantage of amniocentesis is at best minimal in the overall management of these cases

The use of tocolytics is not necessary as the danger that these may cause to the mother and the unborn fetus. However, where ever there is a possibility delivery may be delayed for the administration of the full course of steroids.

This case has raised some important issues about the current management modalities suggested in the literature. Perhaps a more conservative approach might be as good as a more aggressive approach when a holistic view is considered in the management. This case report has highlighted that inflammatory/infective process might play a significant 
role than we currently allude to.

Summary: When the first twin is delivered very prematurely, conservation of pregnancy for undelivered co-twin is beneficial with carful observation of maternal morbidity. Controversy persists as how best to manage very premature multiple pregnancy with delayed delivery interval of the second twins.However, it is well known fact that gestational age at delivery and fetal weight is the most predictor of the good outcome.

\section{Conclusion}

In multiple gestations achieved through assisted reproductive techniques, efforts should be made to prolong such pregnancy following spontaneous miscarriage or premature birth of one of the fetuses. As the association between the interval between the delivery of the first and second twin (intertwin interval) and adverse neonatal outcomes are conflicting. A more conservative approach to treatment might be just as good as the other more invasive approaches reported in the literature.

More prospective studies are needed to assess the long term outcome of such management for both the mother and the baby.

\section{References}

1. B. Blondel and M.Kaminski,"Trends in the occurrence, determinants, and consequences of multiple births," Seminars in Perinatology, vol. 26, no. 4, pp. 239-249, 2002

2. W. F. Powers and J. L. Kiely, "The risks confronting twins: a national perspective," American Journal of Obstetrics and Gynecology, vol. 170, no. 2, pp. 456-461, 199).

3. M. S. To, E. B. Fonseca, F. S. Molina, A. M. Cacho, and K. H. Nicolaides, "Maternal characteristics and cervical length in the prediction of spontaneous early preterm delivery in twins," American
Journal of Obstetrics and Gynecology, vol. 194, no. 5, pp. 1360-1365, 2006.

4. WHO. Preterm birth.

5. Bliss.org.uk. Statistics.

6. Costeloe KL, Hennessy EM, Haider S, et al. Short term outcomes after extreme preterm birth in England: comparison of two birth cohorts in 1995 and 2006(the EPICure studies: BMJ 2012;345:E7976.

7. Intertwin time interval: How it affects the immediate neonatal outcome of the second twin. Caprice D. McGrail, MD David R. Bryant, MDMay 2005Volume 192, Issue 5, Pages 1420-1422

8. The Association between the Intertwin Interval and Adverse Neonatal Outcomes Kate Swanson, William A Grobman, Emily S MillerAmerican Journal of Perinatology 2017, 34 (1): 70-73

9. Alon Tal, Hadar Peretz, Gali Garmi, Noah Zafran, Shabtai Romano, Raed Salim-Effect of intertwin delivery interval on umbilical artery PH and Apgar score in second twin. BIRTH. Volume 45, issue 1 March 2018, pages 37 -42).

10. J. Zhang, C. D. Johnson, and M. Hoffman, “Cervical cerclage in delayed interval delivery in a multifetal pregnancy: a review of seven case series," European Journal of Obstetrics Gynecologyand Reproductive Biology, vol. 108, no. 2, pp. 126130, 2003.

11. Lockwood CJ. The diagnosis of preterm labor and the prediction of preterm delivery. Clin Obstet Gynecol. 1995;38:675-87. [PubMed]

12. Lamont RF. Infection in the prediction and antibiotics in the prevention of spontaneous preterm labour and preterm birth. BJOG. 2003;110(Suppl 20):71-5

13. Goldenberg RL, Hauth JC, Andrews WW. Intrauterine infection and preterm delivery. $\mathrm{N}$ Engl J Med2000;342: 1500 - 1507

14. Hay PE, Lamont RF, Taylor-Robinson D, Morgan DJ, Ison C, Pearson J. Abnormal bacterial coloni- 
sation of the genital tract and subsequent preterm delivery and late miscarriage. BMJ (Clinical research ed).1994;308(6924):295-8.

15. Kurki T, Sivonen A, Renkonen OV, Savia E, Ylikorkala 0 . Bacterial vaginosis in early pregnancy and pregnancy outcome. ObstetGynecol. 1992;80(2):173-7

16. Lamont RF, Nhan-Chang CL, Sobel JD, Workowski $\mathrm{K}$, Conde-Agudelo A, Romero R. Treatment of abnormal vaginal flora in early pregnancy with clin- damycin for the prevention of spontaneous preterm birth: a systematic review and metaanalysis. American journal of obstetrics and gynecology. 2011;205(3):177-90
Received 30-7-2018

Revised 15-9-2018

Accepted 27-9-2018 